\title{
Management of Persistent Perfusion of an Excluded Popliteal Artery Segment Following Penetrating Vascular Injury
}

\author{
CDR Brian S Knipp MD, LCDR Shalimar J Andrews MD, Halley R Werwath RVT \\ and CDR Daniel M Sutton MD
}

Naval Medical Center, Portsmouth, USA

\begin{abstract}
Exclusion and bypass for penetrating vascular injury remains the gold standard. Persistent hemorrhage via retrograde perfusion of the injured vessel by collateral vessels is rare and may, therefore, be overlooked as a cause of postoperative hemorrhage following bypass for vascular injury. We report a case of a 49-year-old male who presented to our institution 2 weeks following a bypass graft of his popliteal artery after sustaining a gunshot wound to the vessel. His ongoing complaints of pain and pressure in the popliteal fossa were found to be related to persistent collateral perfusion of the injured segment of his proximal popliteal artery. His symptoms resolved completely following coil embolization of the injured native artery. While quite rare in clinical practice, the pathophysiology of this case is analogous to persistent perfusion of the aneurysm sac following open abdominal aortic aneurysm repair or bypass and exclusion of a popliteal artery. In the setting of bypass grafting for vascular trauma, postoperative hemorrhage or compressive symptoms should prompt a complete evaluation for a potentially missed patent collateral vessel.
\end{abstract}

Keywords: Peripheral Vascular Trauma; Embolization; Endoleak

Received: 7 December 2020; Accepted: 1 March 2021

\section{INTRODUCTION}

A standard technique for the management of penetrating vascular injury with arterial disruption is exclusion and bypass. While little has been reported in the literature regarding persistent retrograde perfusion of the

\section{Corresponding author:}

Brian S. Knipp, MD, FACS, CDR, Navy Trauma Training Center, 1200 N State Street, Room 1050, Los Angeles, CA 90033, USA. Email: brian.s.knipp.mil@mail.mil

Disclosure: The views expressed in this manuscript are those of the author(s) and do not necessarily reflect the official policy or position of the Department of the Navy, Department of Defense, or the United States Government. B.S. Knipp is a military service member. This work was prepared as part of his official duties. Title 17 U.S.C. 105 provides that "Copyright protection under this title is not available for any work of the United States Government." Title 17 U.S.C. 101 defines a United States Government work as a work prepared by a military service member or employee of the United States Government as part of that person's official duties.

(c) 2021 CC BY 4.0 - in cooperation with Depts. of Cardiothoracic/ Vascular Surgery, General Surgery and Anesthesia, Örebro University Hospital and Örebro University, Sweden injured segment via collateral vessels, this entity is recognized in the pathophysiology of endovascular leaks (endoleaks) following endovascular aneurysm repair (EVAR), branch vessel bleeding into the aneurysm sac following open repair of abdominal aortic aneurysms, and persistent pressurization of the aneurysm sac following treatment of popliteal artery aneurysms (PAA). We present a case of lower extremity compressive symptoms following exclusion and bypass of an injured popliteal artery that resolved immediately and completely following coil embolization of the feeding vessel and residual excluded segment.

\section{CASE REPORT}

Following a gunshot wound to the right distal thigh above the knee, a 49-year-old male presented to the regional trauma center complaining of pain, paresthesia, and motor weakness of the leg. His right femoral pulse was palpable, but there were no palpable pulses or audible Doppler signals in the popliteal or pedal arteries. A computed tomography (CT) scan demonstrated acute occlusion of the proximal popliteal artery with distal reconstitution of the tibial arteries via geniculate 


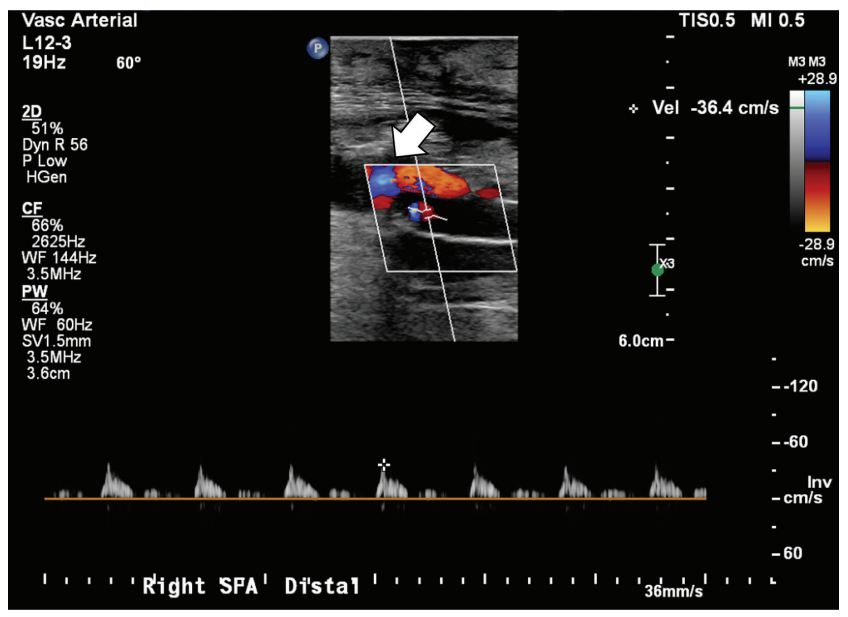

Figure 1 Demonstration of pulsatile flow in the most proximal portion of the excluded popliteal artery (labeled as "Right SFA Distal"). The proximal graft anastomosis is represented by the white arrow.

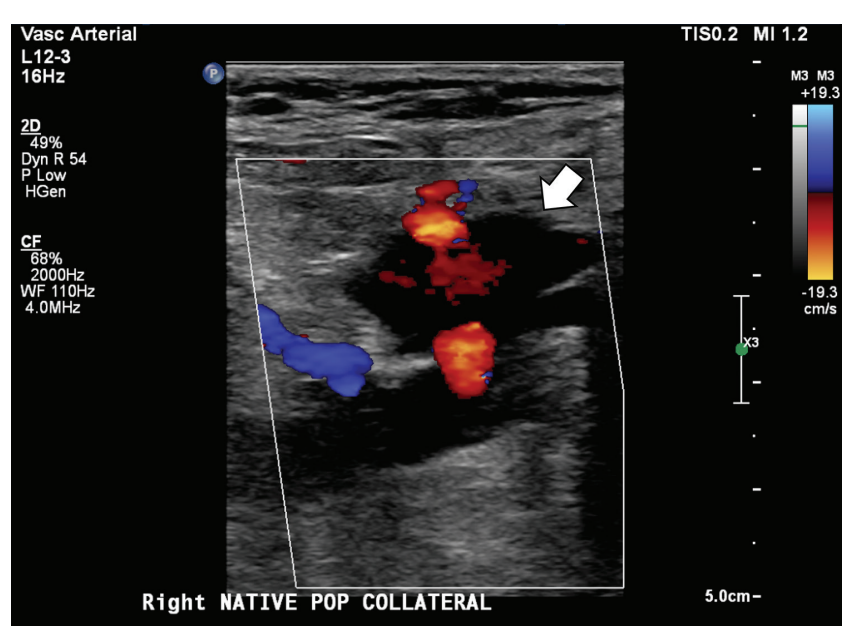

Figure 2 Flow through collaterals is visualized entering into the native popliteal artery as well as an extraluminal fluid collection (arrow).

collaterals. No active contrast extravasation was seen on the CT scan. The patient underwent above-to-below knee popliteal artery bypass grafting using reversed great saphenous vein from the contralateral leg and native artery ligation. A completion arteriogram was performed from the proximal superficial femoral artery, demonstrating a widely patent bypass graft with three-vessel runoff. The pedal pulses were palpable at the end of the procedure. However, the case was complicated by postoperative hemorrhage, requiring two additional explorations, although the source was never identified. The patient was discharged from hospital several days later, still complaining of pain and fullness in the popliteal fossa.

He presented to our military medical center 18 days following the injury with the ongoing complaint of right

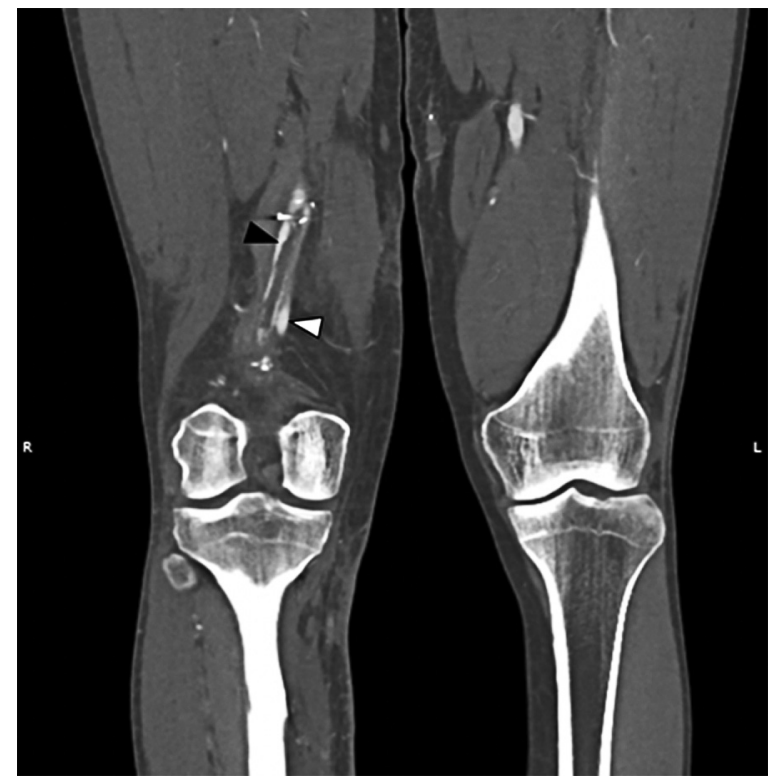

Figure 3 Coronal view of the injured superficial femoral and popliteal artery. Intraluminal thrombus is clearly visualized beyond the proximal vascular clip in the native, "excluded" artery with surrounding contrast (dark arrowhead). The bypass graft is seen only partially as it is mostly out of plane in this view (white arrowhead).

leg pain localized to the region of injury. He reported persistent swelling in the popliteal fossa and pain with ambulation, and stated that he was unable to perform his activities of daily living due to the pain and sense of instability. His physical examination was notable for easily palpable pedal pulses, and his foot was warm and well perfused with normal motor and sensory function. His incisions were well-approximated without evidence of infection. A duplex study, however, demonstrated multiphasic flow within the injured popliteal artery segment, which was perfused by collaterals (Figures 1 and 2). A CT angiogram confirmed these findings; the proximal portion of the surgically excluded popliteal artery was found to be filling on arterial phase contrast imaging (Figures 3 and 4). The underlying pathophysiology seemed to be a popliteal fossa hematoma originating from the injured popliteal artery and under near-systemic arterial pressure via collaterals.

Based on the imaging findings, the patient underwent a right lower extremity arteriogram via left common femoral access. The arteriogram demonstrated a widely patent graft with three-vessel runoff (Figure 5). The excluded segment of the popliteal artery was visualized filling via distal profunda femoris arterial collaterals (Figures 6-8). The profunda femoris artery was selected and a mildly hypertrophied distal branch was subselected. Digital subtraction angiography (DSA) imaging verified this branch filling the excluded segment. Smaller collateral branches extending from the excluded segment were identified supplying soft tissue in the immediate 

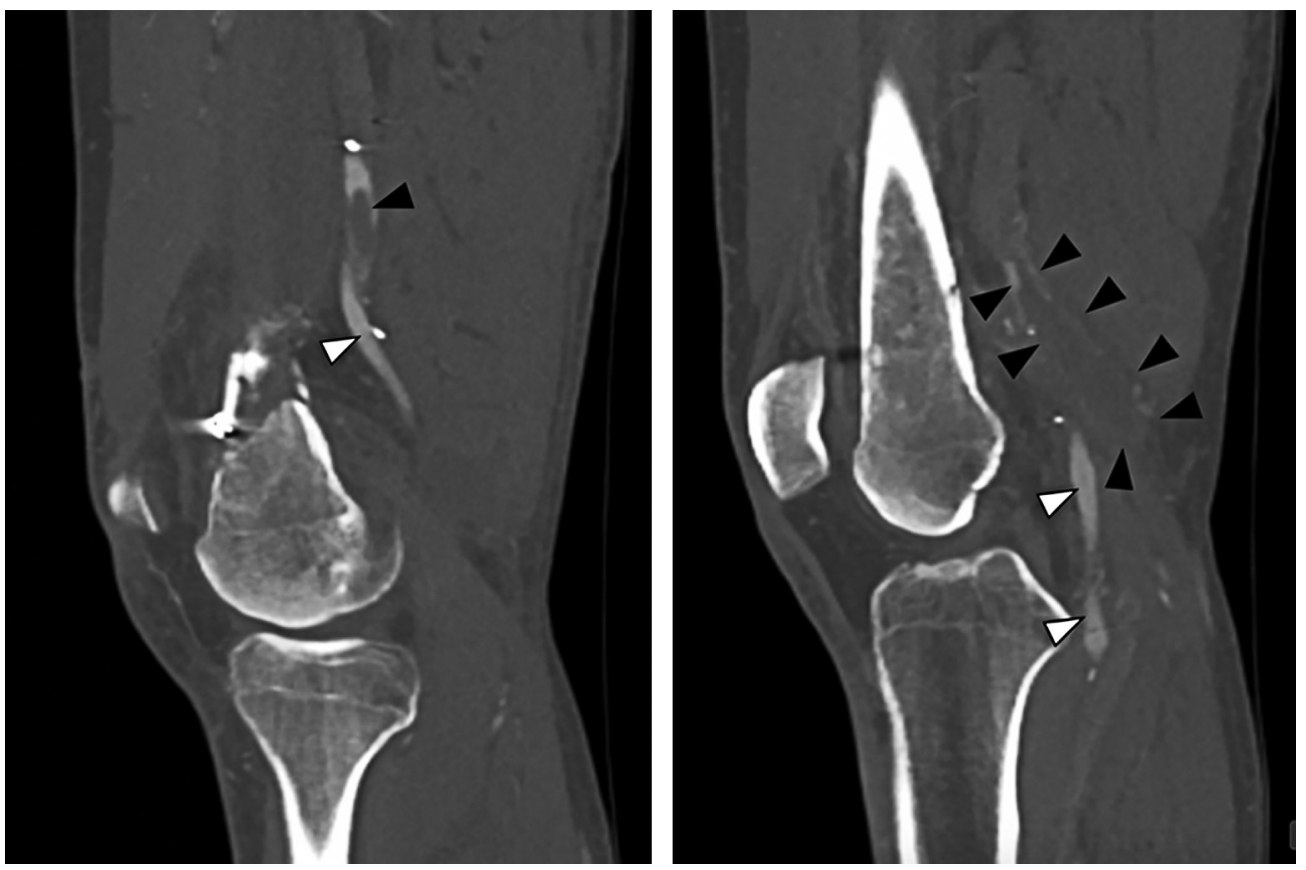

Figure 4 Sagittal view of the native artery with contiguous hematoma in the distal thigh and proximal popliteal fossa (dark arrowheads). The arterial contrast can be seen along the edges of the hematoma. The bypass graft is shown by the white arrowheads.

vicinity. A Progreat microcatheter was manipulated into the excluded arterial segment and approximately 3-5 $\mathrm{ml}$ of dilute 700-900 micron microspheres (Embosphere; Biosphere Medical, Rockland, MA) were injected to decrease the risk of retrograde flow into the excluded segment. These microspheres lodge in the arterial outflow branches above the capillary bed level, thereby limiting the potential for ischemic injury and necrosis of the soft tissues. Immediate post-embolization DSA imaging demonstrated markedly decreased flow through the segment and into the surrounding tissue. Next, multiple Azur CX (Terumo, Somerset, NJ) and VortX (Boston Scientific, Marlborough, MA) metallic coils were deployed (Figures 9 and 10) into the excluded segment and postembolization DSA imaging demonstrated no filling of this excluded segment. The patient noted immediate relief of his symptoms at the conclusion of the procedure. He has been seen multiple times in followup with no recurrence of his symptoms. His bypass graft remains widely patent.

\section{Ethical Approval and Informed Consent}

Ethical approval was not required. The information has been anonymized and informed consent was not required.

\section{DISCUSSION}

In the setting of hemorrhage or compressive symptoms following bypass for vascular trauma, strong consideration must be given to the possibility of an unrecognized patent collateral. The complication of persistent retrograde perfusion of an injured arterial segment following exclusion and bypass for trauma has not been wellreported in the literature. It is, however, physiologically similar to three relatively well-recognized phenomena: post-EVAR type II endoleak, aneurysmal sac hemorrhage following open abdominal aortic aneurysm repair, and persistent geniculate artery perfusion of a PAA following exclusion and bypass.

Blood flow entering an excluded segment of native artery by retrograde flow through a branch vessel (e.g., a type-II endoleak following an EVAR procedure) demonstrates both pulsatility and magnitude similar to systemic blood pressure. In 17 patients undergoing treatment for persistent type-II endoleaks following EVAR, Baum et al. observed pulsatile waveforms in the excluded aneurysm sac within $20 \%$ of systemic pressure in all patients [1]. Following 1218 open abdominal aortic aneurysm repairs, Darling reported 48 cases $(4 \%)$ of persistent flow into the aneurysm sac seen on duplex imaging; in 7 of these cases $(0.5 \%)$, this led to aneurysm sac rupture [2]. Hartung et al. reported a case of persistent retroperitoneal hemorrhage following EVAR repair for a ruptured abdominal aortic aneurysm that resulted in persistent back pain, hypotension, and hemodynamic instability and, subsequently, required coil embolization, leading to a successful outcome [3]. In another case, Sharma et al. presented a case of persistent lumbar branch vessel bleeding 5 months following open repair of a 


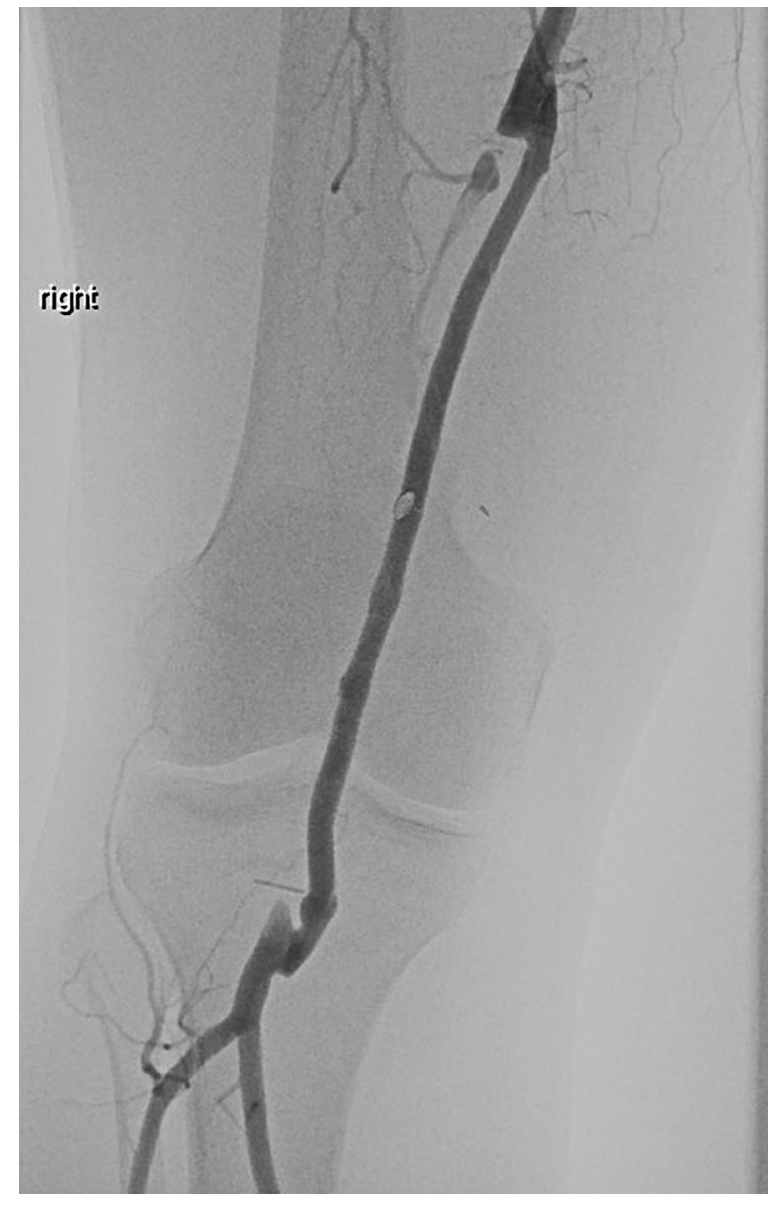

Figure 5 Nonselective right lower extremity angiogram demonstrating persistent filling of the proximal excluded segment of the superficial femoral artery via profunda femoris collaterals.

ruptured aneurysm with associated sac expansion that was successfully treated with Onyx embolization [4].

The other situation analogous to this case is persistent geniculate artery blood flow perfusing a PAA sac following exclusion and bypass. Flynn et al. reported two cases of continued aneurysm expansion with compressive symptoms following exclusion and bypass of PAA due to geniculate side-branch perfusion [5]. In a study of 26 PAAs treated via exclusion and bypass technique with at least 24 months of followup data, there was a $38 \%$ incidence of retrograde flow into the aneurysm sac via geniculate collaterals; $23 \%$ of aneurysms increased in size, and $12 \%$ ruptured [6]. In all cases that underwent posterior sac decompression following a previous exclusion and bypass, the sac demonstrated pulsatile waveforms at nearly systemic pressures. Battey et al. presented a case of a ruptured previously bypassed PAA, demonstrating that the pressures transmitted via geniculate collaterals were sufficient to lead to this outcome. Rupture of a PAA is rare, occurring in only $2-3 \%$ of cases. Rupture of excluded aneurysms is much less common, making this entire pathologic entity extremely rare

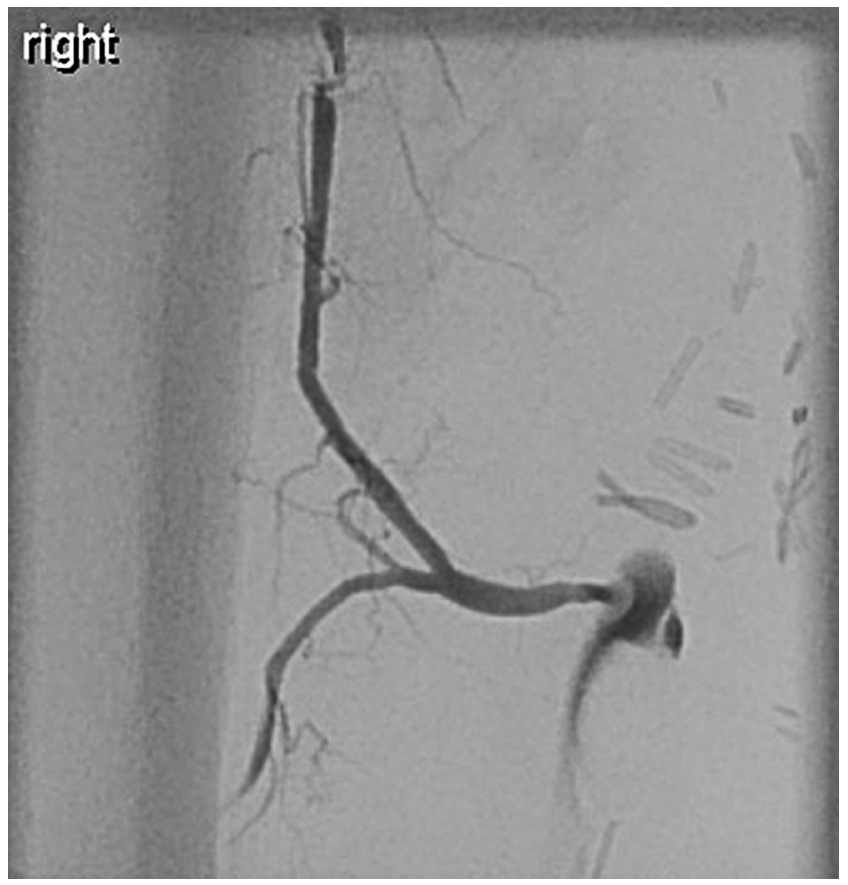

Figure 6 Selective angiogram demonstrating antegrade filling of the injured superficial femoral artery. Note the proximal vascular clip placed at the time of the bypass graft.

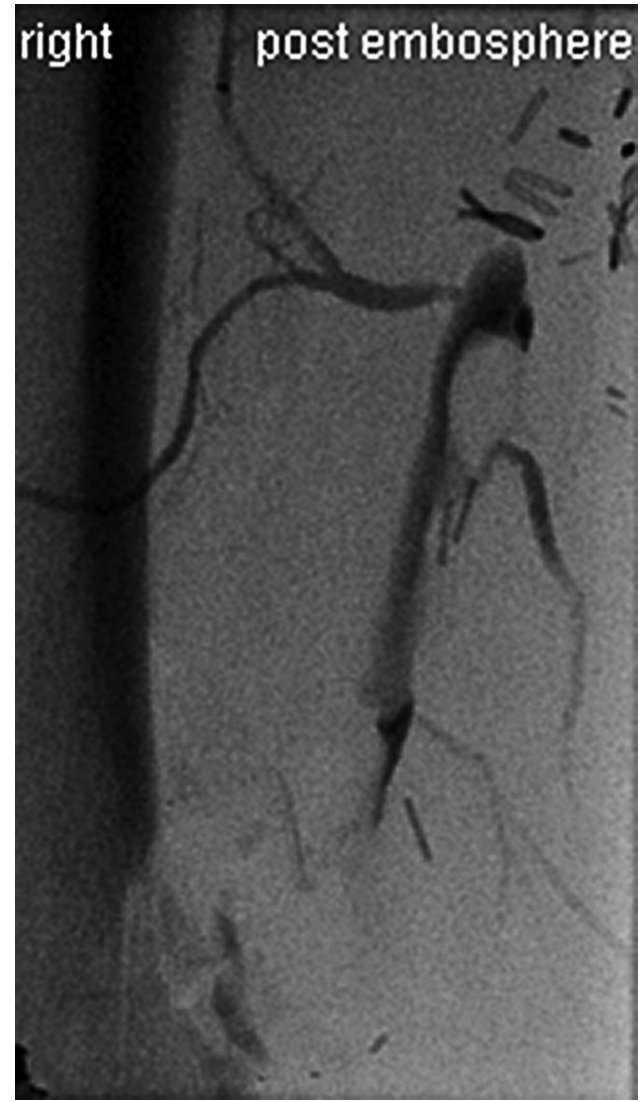

Figure 7 Following the injection of embolospheres, a repeat arteriogram was performed, which demonstrated persistent flow into the native excluded artery with outflow via multiple collaterals as well as contrast extravasation. 


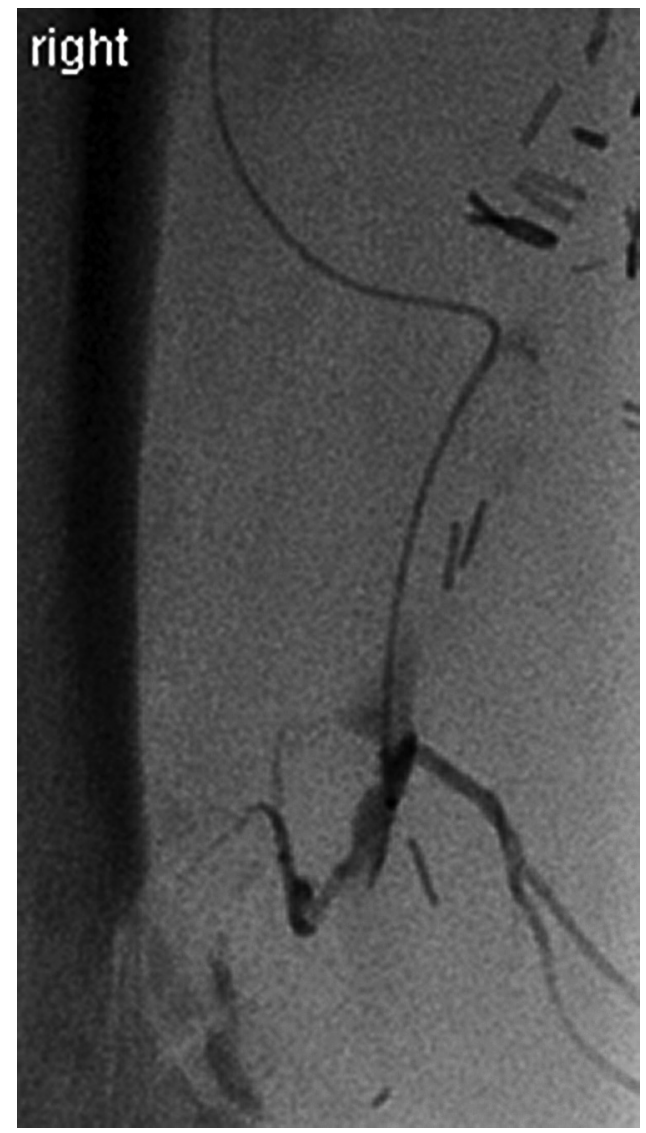

Figure 8 Subselective catheterization of the injured native artery.

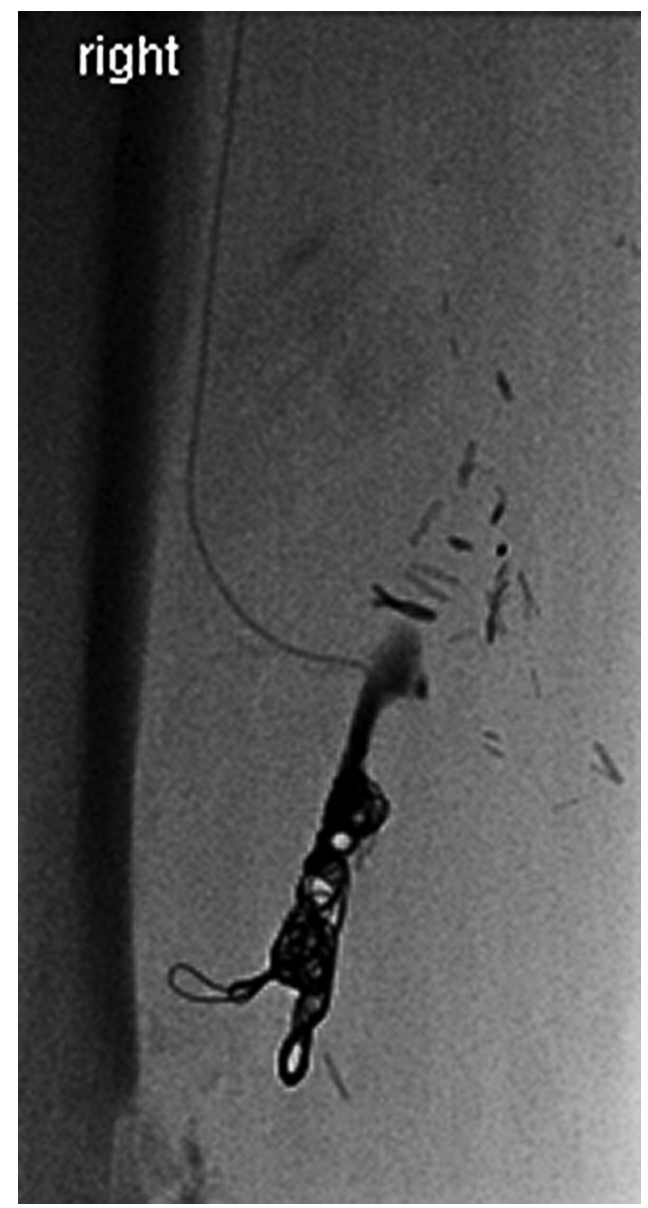

Figure 9 Multiple thrombogenic coils were placed in the excluded segment of the artery. in clinical practice [7]. Bypass and exclusion of the PAA remains the gold standard operation, given decreased complexity vis a vis the posterior approach. Awareness of the potential for retrograde perfusion via geniculate collaterals and early intervention should symptoms develop seems an appropriate tradeoff for the reduced risk of surgical complications [5]. Szilagyi et al. specifically warned about the risk of leaving geniculate collateral vessels unligated. "It must be emphasized, however, that the ligation of the aneurysm must be carefully carried out and no potential collateral arterial branches must be left between the aneurysm and the ligature if a recurrence of the aneurysm is to be prevented [8]."

These same principles would arguably hold in the case of penetrating arterial trauma. While we were unable to find other reports in the literature describing persistent retrograde arterial perfusion from branch vessels into an injured and excluded arterial segment, the situation is directly analogous to type-II endoleak following EVAR, hemorrhage into the aneurysm sac of an open abdominal aortic aneurysm repair, and persistent geniculate collateral flow into an excluded PAA sac with expansion. While all of these entities are uncommon, they are well documented in the literature. Once the pattern is recognized, the solution is straight forwardocclusion of flow via the branch vessel into the excluded arterial segment. While endovascular approaches to the culprit vessel are ideal in settings in which this capability is available, in austere or low-resource environments, open exploration might be necessary. Just as open-sac exploration is the proper solution for persistent type-II endoleaks following EVAR, if catheter-based techniques are either not available or have proven unsuccessful $[9,10]$, then open exploration is the proper course in these resource-constrained environments.

\section{CONCLUSIONS}

Analogous to symptomatic type-II endoleak following EVAR, persistent aneurysm sac bleeding following open abdominal aortic aneurysm repair, and geniculate flow leading to a pressurized excluded PAA sac, the pain and compressive symptoms following exclusion and bypass of an injured native artery should prompt 


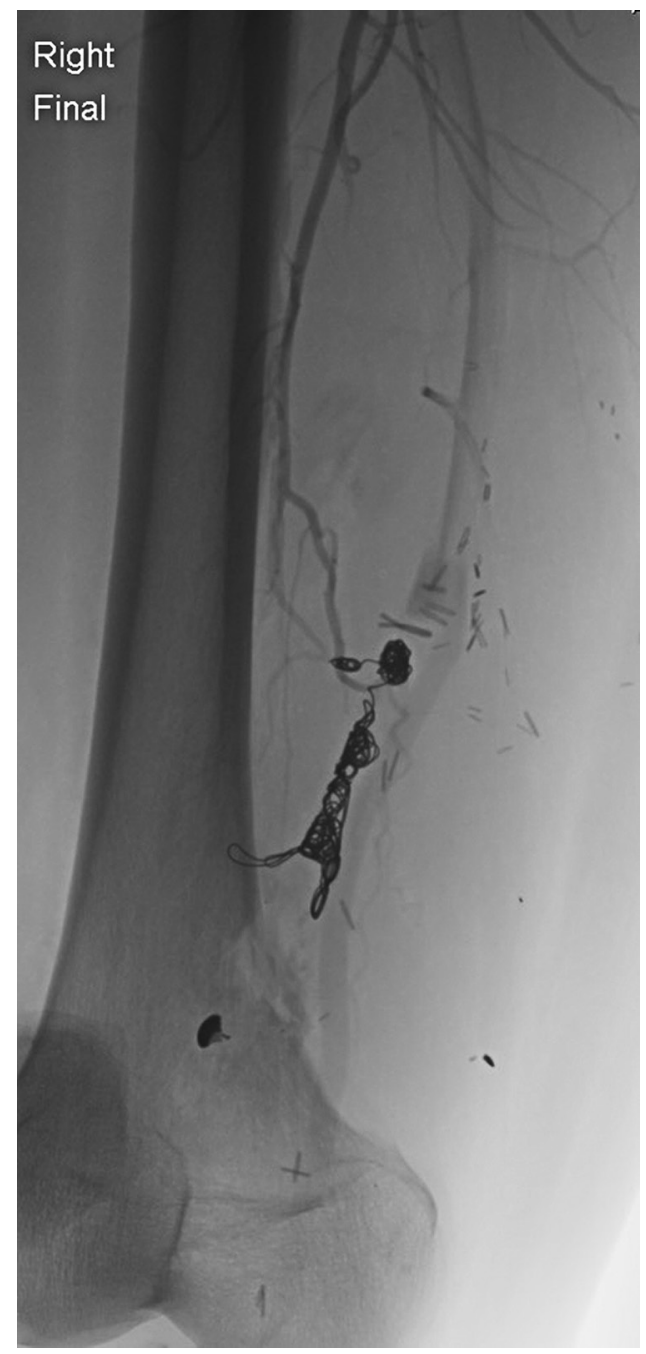

Figure 10 Completion arteriogram which demonstrates complete thrombosis of the native injured segment of the superficial femoral artery with preserved flow through the bypass graft.

an evaluation for a missed patent branch vessel. In addition, duplex ultrasound followup for exclusion and bypass of injured peripheral arteries as well as popliteal aneurysms should include an evaluation of the injured segment to rule out persistent flow into the native segment, a pseudoaneurysm, or a contained hematoma. This is generally overlooked on routine postoperative surveillance studies.

\section{Ethics Statement}

(1) All the authors mentioned in the manuscript have agreed to authorship, read and approved the manuscript, and given consent for submission and subsequent publication of the manuscript.

(2) The authors declare that they have read and abided by the JEVTM statement of ethical standards including rules of informed consent and ethical committee approval as stated in the article.

\section{Conflict of Interest}

The authors declare that there are no conflicts of interest to disclose.

\section{Funding}

The authors received no financial support for the research, authorship, and/or publication of this article.

\section{REFERENCES}

[1] Baum RA, Carpenter JP, Cope C, et al. Aneurysm sac pressure measurements after endovascular repair of abdominal aortic aneurysms. J Vasc Surg. 2001;33(1):32-41.

[2] Darling RC, 3rd, Ozsvath K, Chang BB, et al. The incidence, natural history, and outcome of secondary intervention for persistent collateral flow in the excluded abdominal aortic aneurysm. J Vasc Surg. 1999;30(6):968-76.

[3] Hartung O, Vidal V, Marani I, Saran A, Bartoli JM, Alimi YS. Treatment of an early type II endoleak causing hemorrhage after endovascular aneurysm repair for ruptured abdominal aortic aneurysm. J Vasc Surg. 2007; 45(5):1062-5.

[4] Sharma K, Halandras P, Milner R. Atypical presentation of a type 2 endoleak following emergency open repair of a ruptured abdominal aortic aneurysm. EJVES Short Rep. 2016;33:24-6.

[5] Flynn JB, Nicholas GG. An unusual complication of bypassed popliteal aneurysms. Arch Surg. 1983;118(1): 111-3.

[6] Mehta M, Champagne B, Darling RC, 3rd, et al. Outcome of popliteal artery aneurysms after exclusion and bypass: significance of residual patent branches mimicking type II endoleaks. J Vasc Surg. 2004;40(5):886-90.

[7] Battey PM, Skardasis GM, McKinnon WM. Rupture of a previously bypassed popliteal aneurysm: a case report. J Vasc Surg. 1987;5(6):874-5.

[8] Szilagyi DE, Schwartz RL, Reddy DJ. Popliteal arterial aneurysms. Their natural history and management. Arch Surg. 1981;116(5):724-8.

[9] Kelly A, Toale C, Power D, Kavanagh EG, Moloney MA. Open repair of a type II endoleak facilitated by temporary disconnection of graft components. J VascSurg Cases Innov Tech. 2021;7(1):26-9.

[10] Yamada M, Takahashi H, Tauchi Y, Satoh H, Matsuda H. Open surgical repair can be one option for the treatment of persistent type II endoleak after EVAR. Ann Vasc Dis. 2015;8(3):210-4. 